\title{
Tratamento endovascular de síndrome congestiva venosa pélvica em paciente com duplicação de veia cava inferior e anatomia venosa pélvica incomum: revisão bibliográfica
}

\author{
Endovascular treatment of pelvic venous congestion syndrome in a patient with \\ duplication of the inferior vena cava and unusual pelvic venous anatomy: literature review \\ Marcelo Fernandes Lima (D), llídio Almeida Lima² (D), Vanessa Heinrich-Oliveira ${ }^{3}$
}

\begin{abstract}
Resumo
A dor pélvica crônica afeta aproximadamente $1 / 3$ de todas as mulheres e é responsável por cerca de $20 \%$ de todas as consultas ginecológicas. Os autores relatam um raro caso de congestão venosa pélvica sintomática na presença de duplicação de veia cava inferior e comunicação interilíaca através de veia hipogástrica direita tratado com abordagem endovascular, por embolização das veias varicosas pélvicas e revisão da literatura publicada.
\end{abstract}

Palavras-chave: congestão venosa pélvica; veia cava inferior duplicada; varizes pélvicas.

\begin{abstract}
Chronic pelvic pain affects approximately one-third of all women and is responsible for about $20 \%$ of all gynecological consultations. The authors report a rare case of symptomatic pelvic venous congestion in the presence of duplication of the inferior vena cava and inter-iliac communication through the right hypogastric vein that was treated via an endovascular approach with embolization of varicose pelvic veins. The published literature is reviewed.
\end{abstract}

Keywords: pelvic venous congestion; inferior vena cava duplication; pelvic varicose veins.

Como citar: Lima MF, Lima IA, Heinrich-Oliveira V. Tratamento endovascular de síndrome congestiva venosa pélvica em paciente com duplicação de veia cava inferior e anatomia venosa pélvica incomum: revisão bibliográfica. J Vasc Bras. 2020;19:e20190017. https://doi.org/10.1590/1677-5449.190017 


\section{INTRODUÇÃO}

A dor pélvica crônica (DPC) é definida como dor em região pélvica, não cíclica, por tempo superior a 3 meses. Ela acomete cerca de um 1/3 de todas as mulheres, sendo esse sintoma responsável por até $20 \%$ de todas as consultas ginecológicas. Entre as principais etiologias de DPC, estão endometriose, adenomiose, doença inflamatória pélvica e leiomiomas ${ }^{1}$. Quando veias varicosas se desenvolvem em torno dos vários órgãos pélvicos, desenvolve-se um quadro de congestão venosa pélvica (CVP) que pode levar a um cenário de DPC. ACVP é mais frequentemente diagnosticada em mulheres multíparas, com quadro clínico tipicamente caracterizado por dor abdominal baixa ou pélvica não cíclica, exacerbada por ortostatismo prolongado, relação sexual, no período menstrual e na gravidez. A dor é caracterizada como tipo peso, com sintomas associados, como cefaleia, inchaço, náuseas, peso em membro inferior, dor lombar, desconforto retal, urência urinária, letargia e depressão. O conjunto de sintomas associado ao achado no exame físico de varizes em vulvas, períneo, face posterior de raiz de membros inferiores e nádegas é forte indicador da presença de CVP, devendo ser procedida a investigação para confirmação diagnóstica com métodos de imagens adequados $^{2}$. O primeiro relato de duplicação de veia cava inferior (VCI) foi feito em 1916, em Londres (A case of double inferior vena cava. Lucas MF. J Anat $1916 ; 51: 69-70)^{3}$. Sua incidência vem sendo estimada, desde então, entre 0,3 e 3\% em vários relatos, com a grande maioria dos casos sendo assintomática, com diagnóstico incidental. No entanto, o conhecimento das variações anatômicas da VCI é de vital importância, especialmente durante cirurgias retroperitoniais e intervenções endovasculares ${ }^{3}$.

Apesar da baixa qualidade, devido ao desenho inadequado, dos estudos disponíveis avaliando a eficácia do tratamento endovascular com oclusão das veias varicosas com molas e/ou injeção de substâncias esclerosantes ${ }^{4}$, a abordagem endovascular com embolização das veias varicosas pélvicas e pontos de refluxo parece ser o melhor método de tratamento na atualidade para a $\mathrm{CVP}^{5}$. O caráter minimamente invasivo dos procedimentos endovasculares permite o tratamento desses pacientes em um regime ambulatorial ou de hospital-dia, reduzindo tanto o desconforto quanto os custos de um procedimento cirúrgico convencional. $\mathrm{O}$ sucesso terapêutico das embolizações para tratamento da CVP relatado varia entre 70 e $85 \%$, sem impactos negativos no ciclo menstrual, fertilidade ou níveis hormonais ovarianos, com taxa de complicações estimada entre 3,4 e $9 \%{ }^{6}$.

\section{RELATO DE CASO}

A presente revisão bibliográfica foi motivada após atendimento de uma paciente do sexo feminino, nuligesta, 27 anos de idade, que procurou assistência queixando-se de dor urente, peso e cansaço em membros inferiores, sintomas esses exacerbados com piora durante o período menstrual. Ao exame físico da paciente, observou-se a presença de veia varicosa calibrosa em face póstero-medial de terço superior de coxa esquerda, além de varículas e telangiectasias distribuídas por ambos os membros inferiores. À anamnese, revelou queixas compatíveis com CVP, como dispareunia e dor pélvica de forte intensidade durante o período menstrual, bem como infecções urinárias de repetição.

A investigação com exames de imagens foi feita com duplex scan venoso de membros inferiores, que não evidenciou alterações significativas nos sistemas venosos superficiais e profundos, e com uma angiotomografia de fase venosa, que confirmou a presença de veias varicosas pélvicas, além de evidenciar VCI infrarrenal duplicada, que formava conduto único a partir da desembocadura da veia renal esquerda (Figuras 1 e 2).

A estratégia terapêutica traçada foi a eliminação dos pontos de refluxo proximais por embolização percutânea das veias varicosas pélvicas com implante

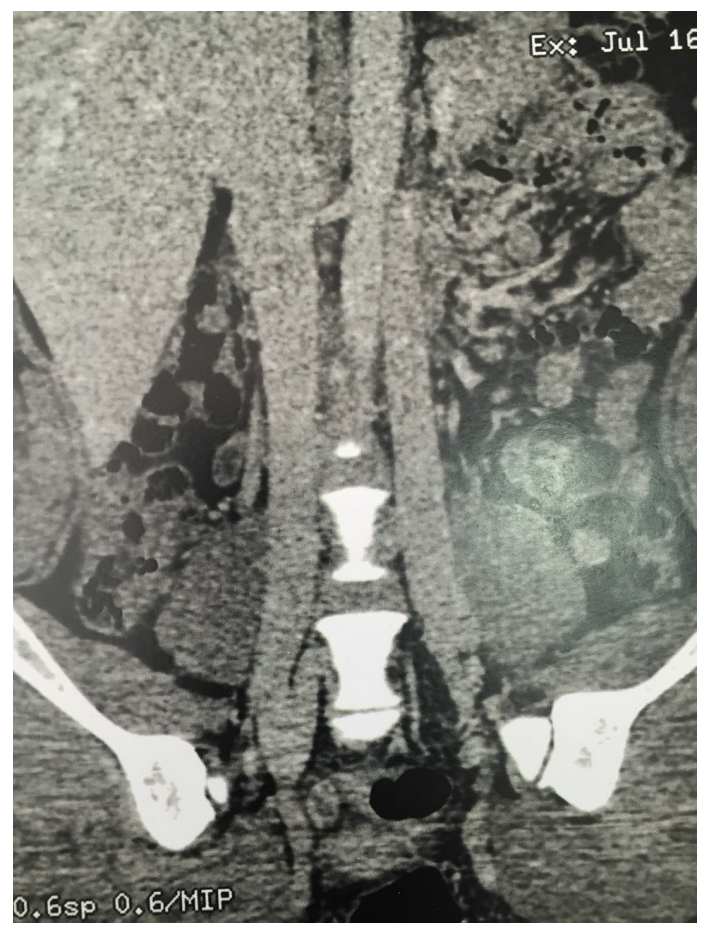

Figura 1. Angiotomografia de fase venosa mostrando duplicidade de veia cava inferior, formando conduto único a partir de desembocadura de veia renal esquerda. 
de molas de platina fibradas e posterior escleroterapia química das veias varicosas dos membros inferiores. A etapa inicial do tratamento foi realizada por acesso percutâneo de veia femoral esquerda, com flebografia pélvica e abdominal confirmando a duplicidade da VCI, com cada veia ilíaca comum drenando para a respectiva cava inferior (Figuras 3 e 4), bem como a

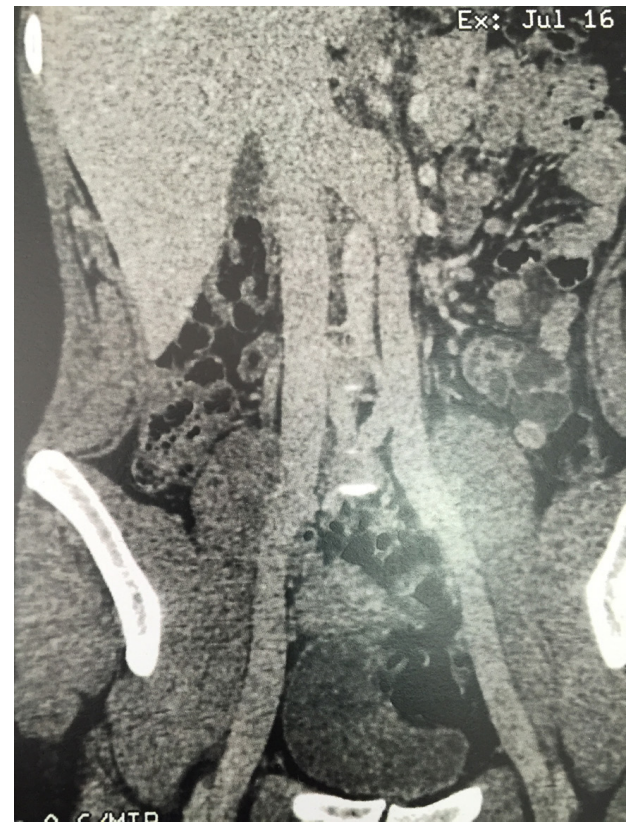

Figura 2. Angiotomografia de fase venosa mostrando duplicidade de veia cava inferior, formando conduto único a partir de desembocadura de veia renal esquerda.

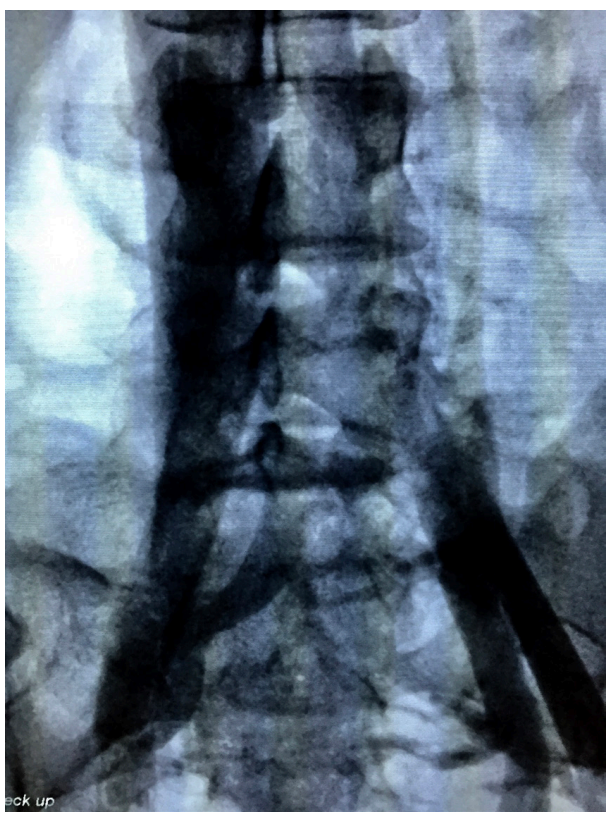

Figura 3. Angiografia mostrando a presença de sistemas ilíacos independentes, com presença de veia interilíaca. presença de veia interilíaca comunicando o sistema ilíaco direito ao esquerdo, com grande quantidade de varizes pélvicas calibrosas (Figura 5). Procedeu-se, então, a cateterização seletiva da veia interilíaca com acesso ao sistema ilíaco direito (Figura 6), injeção

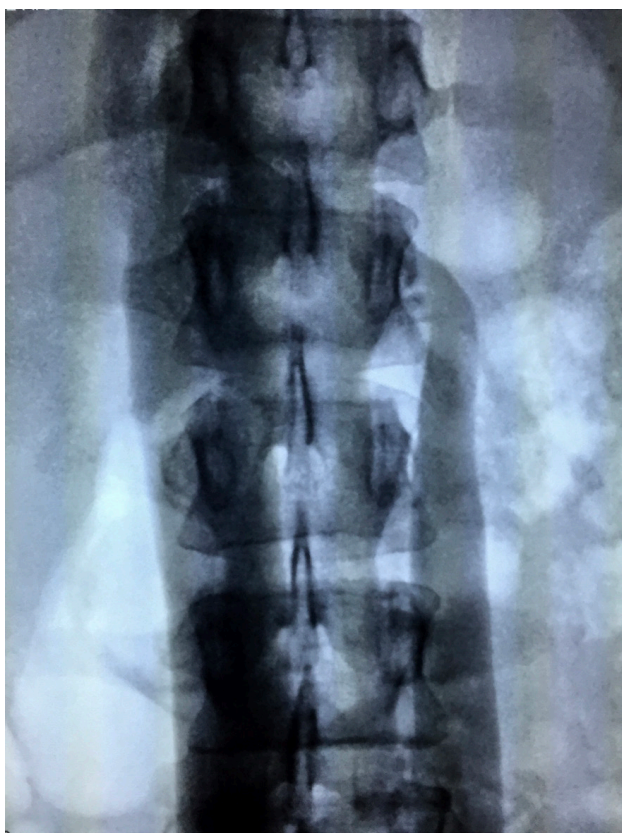

Figura 4. Formação de conduto venoso único com junção das duas veias cavas inferiores após desembocadura de veia renal esquerda.

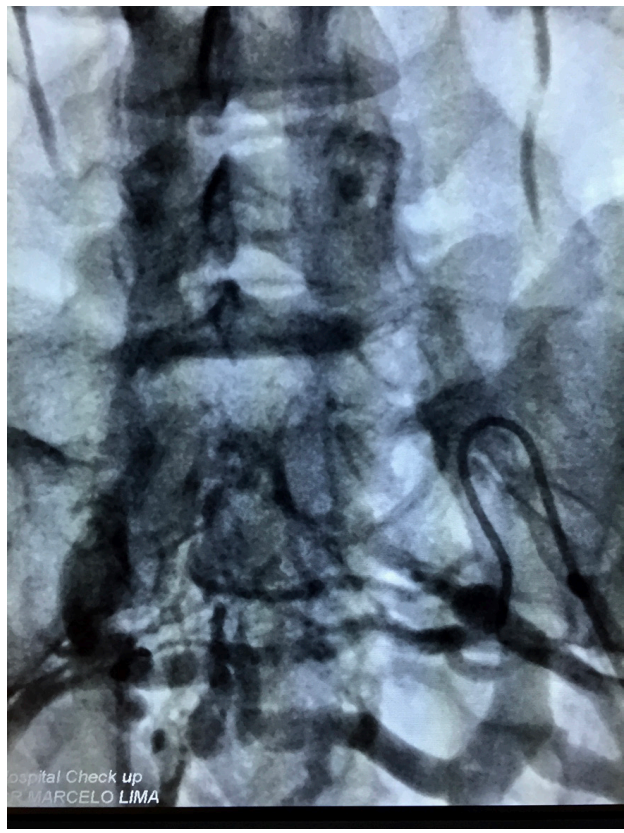

Figura 5. Presença de veia interilíaca comunicando o sistema ilíaco direito ao esquerdo, com grande quantidade de varizes pélvicas calibrosas. 
seletiva de espuma de polidocanol a $1 \%$ em veias varicosas e implante de molas de platina fibradas nos troncos venosos que nutriam as veias varicosas oriundas do sistema venoso ilíaco interno direito (Figura 7). Depois, procedeu-se o acesso seletivo de veia hipogástrica esquerda, com cateterização superseletiva dos plexos venosos varicosos, nova injeção de espuma de polidocanol e implante de molas de platina fibradas também nos troncos venosos nutridores das varizes oriundas do sistema venoso ilíaco interno esquerdo (Figuras 8, 9 e 10). O pós-operatório imediato da paciente transcorreu sem

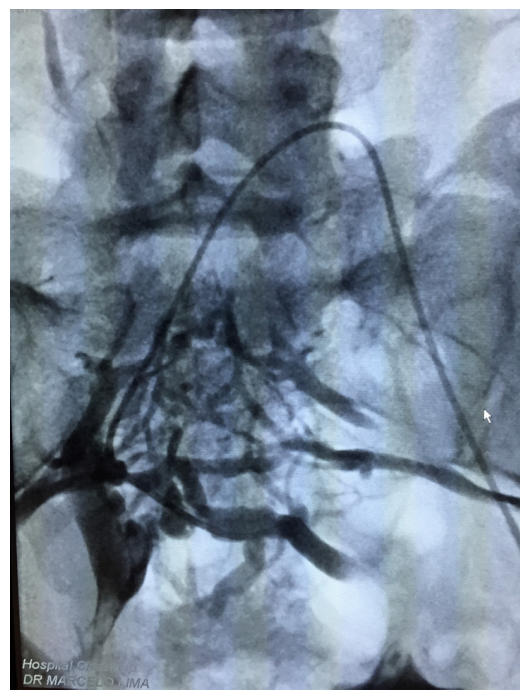

Figura 6. Cateterização seletiva da veia interilíaca com acesso ao sistema ilíaco direito.

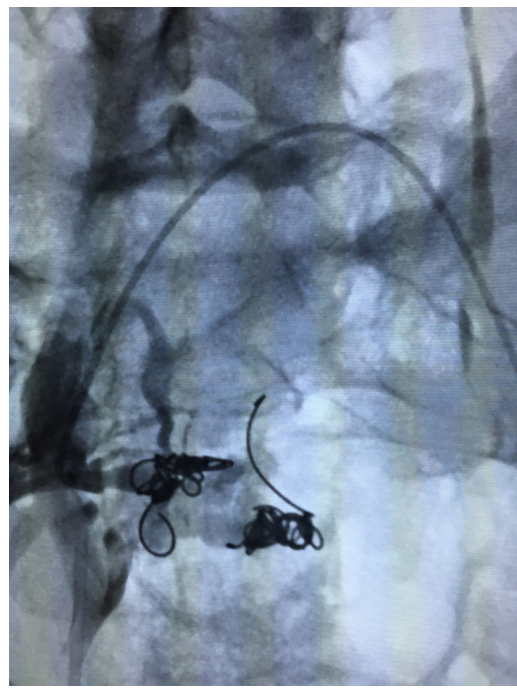

Figura 7. Implante de molas fibradas em veias tributárias do sistema venoso ilíaco direito após injeção de espuma de polidocanol a $1 \%$.

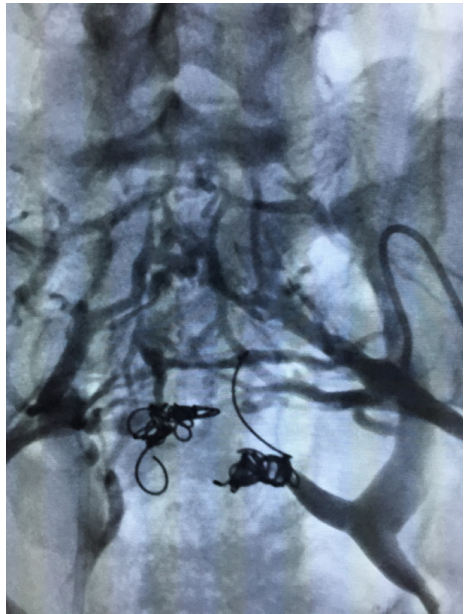

Figura 8. Cateterização seletiva dos troncos venosos nutridores das varizes oriundas do sistema venoso ilíaco interno esquerdo.

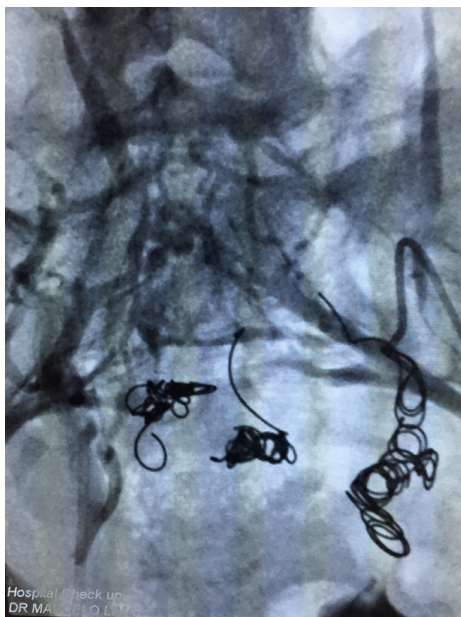

Figura 9. Implante de molas fibradas em veias tributárias do sistema venoso ilíaco esquerdo após injeção de espuma de polidocanol a $1 \%$.

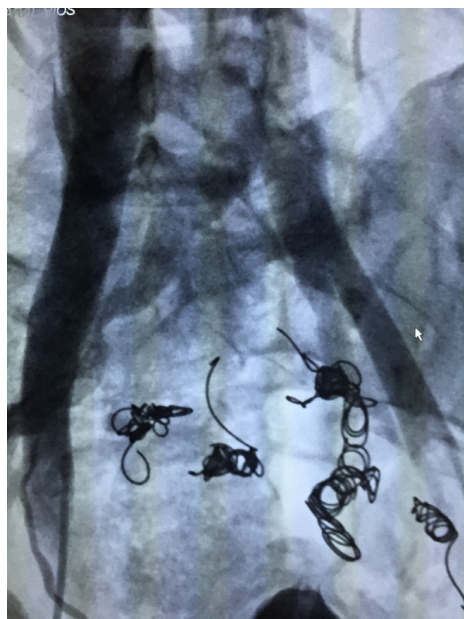

Figura 10. Angiografia de controle após o término do procedimento evidenciando oclusão das veias varicosas pélvicas. 
maiores intercorrências, com queixa de dor moderada pélvica, que cedeu prontamente com administração de analgesia parenteral.

A paciente relatou cessação dos sintomas após a embolização dos plexos venosos varicosos pélvicos, permanecendo assintomática em seguimento após 30 meses da intervenção.

\section{DISCUSSÃO}

A primeira associação entre veias incompetentes e dilatadas na pelve com sintomas de CVP foi feita em 1949, por Taylor. Desde então, não houve publicação de estudos adequados correlacionando a CVP com a DPC ${ }^{7}$. A caracterização clínica de DPC se dá com o quadro de dor contínua ou recorrente, localizada em hipogástrio ou pelve, com duração de pelo menos 6 meses, não limitada a qualquer período do ciclo menstrual ou intercurso, nem associada com gestação. É um importante fator de redução de qualidade de vida e incapacidade laborativa, associada com um significativo fardo mental, social e físico, permanecendo sua etiologia não esclarecida entre 40 e $60 \%$ dos casos, mesmo após estudos de imagem e laparoscopia ${ }^{6,7}$.

Varicosidades pélvicas e DPC são achados típicos da CVP, no entanto, mulheres diagnosticadas com varizes pélvicas podem ser assintomáticas, o que caracteriza um desafio diagnóstico para o ginecologista investigando $\mathrm{DPC}^{6,8}$. A multiparidade é uma constante em pacientes com CVP, com queixas de dismenorreia e exacerbação dos sintomas durante ou após o coito e com ortostatismo prolongado. A origem da CVP é, muito provavelmente, multifatorial, com dois fatores parecendo ter importante papel na gênese do quadro. Primeiro, a incompetência valvular em decorrência de ausência congênita de válvulas ou presença de válvulas defeituosas e, segundo, o aumento em torno de 60 vezes da capacidade venosa pélvica durante a gestação pela compressão mecânica pelo útero gravídico e pela ação vasodilatadora da progesterona, o que pode levar à incompetência de válvulas venosas, com subsequente hipertensão venosa e fluxo retrógrado ${ }^{2,9}$.

Estima-se que $50 \%$ dos pacientes com veias varicosas tenham algum componente genético predisponente. O gene $\mathrm{FOXC} 2$ foi o primeiro a ser implicado na etiologia das veias varicosas e tem um papel-chave no desenvolvimento e funcionamento das válvulas venosas. Outros estudos descobriram associações entre o desenvolvimento de veias varicosas e mutações no TIE2, NOTCH3, trombomodulina e receptor do transformador do fator $\beta$ de crescimento, o que sugere um componente genético para a doença venosa associada com CVP. Outro fator que também pode aumentar a pressão venosa e causar refluxo com aumento do retorno venoso pélvico via colaterais é a compressão mecânica de veias de drenagem, incluindo a síndrome de quebra-nozes, síndrome de May-Thurner, endometriose, fibromas, aderências pós-operatórias, leiomiomas uterinos, tumores ovarianos, mola hidatiforme e tumores mesentéricos. As variações anatômicas da rede venosa pélvica também podem contribuir para o desenvolvimento de $\mathrm{CVP}^{2}$.

As variações anatômicas congênitas da $\mathrm{VCI}$ têm sido cada vez mais relatadas em pacientes assintomáticos em decorrência dos avanços em estudos de imagem. A variação mais comum é a duplicidade de VCI, seguida pela VCI esquerda e continuação da ázigos. No entanto, as variantes anatômicas pélvicas dessas anomalias e suas relações com as veias ilíacas e comunicantes interilíacas não têm merecido a devida atenção, sendo vital o conhecimento dessas para a redução do risco cirúrgico e para determinar estratégias de abordagem em procedimentos de radiologia intervencionista ${ }^{10}$.

A embriogênese da VCI é um processo complexo envolvendo o desenvolvimento, a regressão, a anastomose e a substituição das três principais veias embrionárias. A teoria mais comumente aceita, que tenta explicar a embriogênese da VCI, é a base da Terminologia Embriológica. De acordo com ela, existem três pares de veias embrionárias com várias anastomoses entre elas que regridem e formam a VCI. São elas: as veias cardinais posteriores, as subcardinais e as supracadinais ${ }^{11}$.

A embriogênese da VCI ocorre entre a $4^{\mathrm{a}}$ e a $8^{\mathrm{a}}$ semana de gestação. Nesse ponto, existem três conjuntos de veias pareadas: as supracardinais, as cardinais posteriores e as subcardinais. Essas veias se fundem e regridem sucessivamente, até que a VCI esteja formada ${ }^{12}$. A veia cardinal posterior surge primeiro, na parte posterior do embrião. Essas veias regridem, exceto na porção distal, levando à formação da bifurcação ilíaca. Então, aparecem as veias subcardinais, anterior e medialmente às veias cardinais posteriores. A veia subcardinal direita permanece para formar a VCI suprarrenal, enquanto que a esquerda regride completamente. Subsequentemente, as veias supracardinais aparecem dorsalmente às subcardinais. A supracardinal esquerda regride, e a direita forma a VCI infrarrenal. A VCI normal é convertida, então, num conduto único, unilateral, posicionado à direita, consistindo em quatro componentes: 1) segmento infrarrenal, pela veia supracardinal direita; 2) segmento renal, pela anastomose da supracardinal direita; 3) segmento suprarrenal, pela subcardinal direita; e 4) segmento hepático, pela hepática direita. Várias anastomoses se desenvolvem entre os vários pares de veias cardinais, com o lado direito sendo progressivamente dominante. As anastomoses se formam entre as duas 
veias supracardinais, remanescentes da extremidade distal das veias cardinais posteriores, que formam a veia ilíaca comum esquerda no seu segmento caudal. Os sistemas supracardinais e subcardinais da direita produzem o segmento renal da $\mathrm{VCI}$ e as veias renais ${ }^{13}$. Portanto, a VCI dupla é considerada ser formada pela persistência de ambas as veias supracardinais ${ }^{3}$.

Tão importante quanto avaliar previamente as variações anatômicas da VCI é o conhecimento das inúmeras variações da anatomia venosa pélvica para diminuição do potencial iatrogênico e para determinar estratégias de abordagem em procedimentos de radiologia intervencionista ${ }^{10}$.

A formação das veias ilíacas, incluindo as comunicantes, durante o estágio inicial embrionário é complexa e permanece incerta, sendo relatado que a veia ilíaca interna e suas numerosas tributárias viscerais e parietais servem como importante via colateral nos casos de obstrução segmentar ilíaco-caval. Considerando a variedade de descrições na literatura, o desenvolvimento do sistema das veias ilíacas pode não ser consistente, particularmente em relação à origem primordial das veias hipogástricas ${ }^{14}$.

No intuito de padronizar as diferentes classificações das variações anatômicas da VCI e ilíacas, Morita et al. ${ }^{10}$ revisaram 11.719 estudos tomográficos abdominais e pélvicos em 6.294 pacientes entre janeiro de 2004 e outubro de 2006, encontrando 28 casos de duplicidade VCI, 6 de VCI à esquerda, 1 continuidade de ázigos e uma ausência de VCI infrarrenal. Com base nos achados tomográficos, os autores propuseram a seguinte classificação das variações pélvicas nas anomalias da VCI, sendo definida como veia comunicante interilíaca uma veia que drena sangue das veias ilíacas, incluindo ilíacas comum, externa e hipogástricas, para o lado contralateral da VCI duplicada $^{10}$ (Figura 11):

Tipo 1: confluência ilíaca normal (incluindo a continuação da ázigos);

Tipo 2a: duplicidade da VCI, sem comunicação interilíaca;

Tipo 2b: duplicidade da VCI, com comunicação interilíaca a partir da veia ilíaca comum esquerda;

Tipo 2c: duplicidade da VCI, com comunicação interilíaca a partir da veia ilíaca comum direita;

Tipo 2d: duplicidade da VCI, com comunicação interilíaca a partir da veia ilíaca interna esquerda;

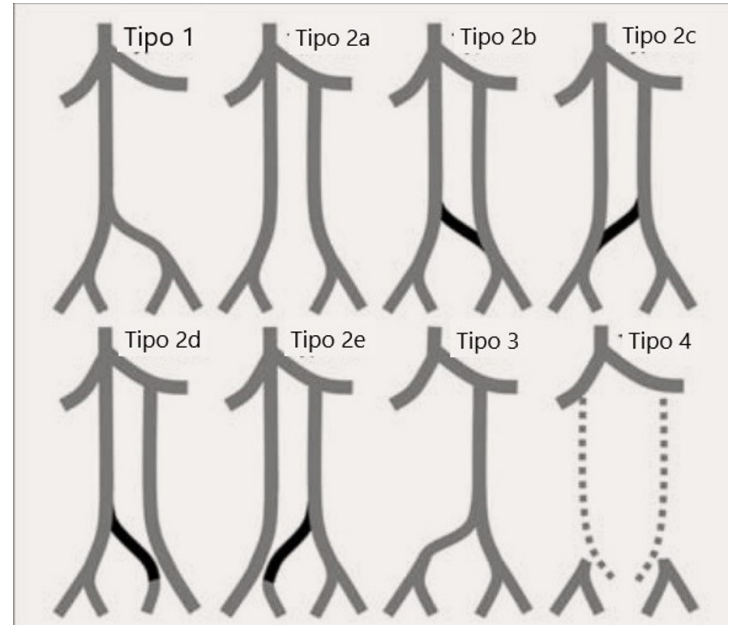

Figura 11. Classificação de Morita et al. ${ }^{10}$ das variações pélvicas nas anomalias da veia cava inferior.

Tipo 2e: duplicidade da VCI, com comunicação interilíaca a partir da veia ilíaca interna direita;

Tipo 3: VCI esquerda com confluência normal ou simétrica das ilíacas;

Tipo 4: sem conjunção ilíaca, com ausência de VCI infrarrenal;

Posteriormente, Hayashi et al. ${ }^{13}$ propuseram uma classificação baseada nos padrões de fluxo das veias ilíacas de acordo com o trajeto das veias hipogástricas, dividindo em três tipos esses padrões:

Tipo L: veia hipogástrica drenando na veia ilíaca externa ipsilateral;

Tipo M: veia comunicante interilíaca;

Tipo S: confluência da veia ilíaca externa ipsilateral e VCI.

A veia cava duplicada, junto com outras anomalias vasculares no retroperitônio, pode ser causa tanto de erro diagnóstico quanto de complicações cirúrgicas. Cirurgiões, radiologistas, oncologistas e urologistas envolvidos no manejo terapêutico de patologias do retroperitônio devem não só ter conhecimento profundo da anatomia normal dessa região, bem como de suas potenciais variantes anatômicas ${ }^{11}$. Zhu Tong et al. ${ }^{15}$ relataram dois casos de implante de filtro de veia cava que não foram eficazes em prevenir a embolização pulmonar pelo desconhecimento da duplicidade de VCI nos pacientes ${ }^{15}$. No nosso meio, Malgor et al. ${ }^{16}$ relataram um implante de filtro de VCI em posição suprarrenal, após a identificação em cavografia da 
duplicidade do vaso, filtro esse que se mostrou eficaz na prevenção da embolia pulmonar ${ }^{16}$.

Embora vários métodos diagnósticos venham sendo avaliados para identificar e diagnosticar a CVP, como ultrassom transvaginal, tomografia computadorizada e ressonância magnética ${ }^{4,6}$, a angiografia permanece como padrão-ouro para o diagnóstico tanto da CVP quanto das anomalias anatômicas da VCI e ilíacas por permitir avaliar os diferentes padrões de fluxo e também o tratamento por embolização com molas ou descompressão de troncos venosos por implante de stents vasculares no mesmo ato ${ }^{4,8,17}$. Alguns critérios angiográficos devem estar presentes para o diagnóstico da CVP, a saber: refluxo demonstrado por injeção proximal de contraste na veia ovariana com enchimento do plexo venoso ovariano distal, veias pélvicas incompetentes com 5 a $10 \mathrm{~mm}$ de diâmetro, estase do fluxo no plexo venoso ovariano, com visualização de veias pélvicas na linha mediana, vulvovaginais e proximais de $\operatorname{coxas}^{6,17}$.

Não há tratamento padronizado para a CVP. As diversas modalidades terapêuticas devem ser individualizadas com base nos sintomas e nas necessidades de cada paciente $^{6}$. Os tratamentos sugeridos para a CVP incluem histerectomia abdominal total, ligadura ou oclusão de varizes pélvicas e terapia hormonal. $\mathrm{O}$ acetato de medroxiprogesterona tem mostrado aliviar temporariamente os escores de dor, mas foi associado a efeitos colaterais como ganho de peso e acne. A ligadura venosa pélvica é raramente realizada atualmente, e a histerectomia total abdominal é inaceitável em mulheres jovens?

Em contraste com os procedimentos cirúrgicos convencionais, os procedimentos endovasculares venosos são minimamente invasivos e conseguem eliminar os pontos de refluxo e as veias varicosas. Geralmente, eles são realizados com anestesia local e/ou sedação venosa, podendo ser em caráter ambulatorial ou em regime de hospital-dia, reduzindo o desconforto, a ansiedade do paciente e os $\operatorname{custos}^{6}$. Atualmente, o método de escolha para tratamento da CVP parece ser a embolização percutânea. Embora tenha-se sugerido que a escleroterapia com espuma seja suficiente para tratar a CVP, poucos dados e evidências corroboram isso. $\mathrm{O}$ implante de molas fibradas nos pontos de refluxo venoso parece ser o padrão-ouro no tratamento endovascular da $\mathrm{CVP}^{5}$.

Com o objetivo de comparar os resultados entre diferentes dispositivos oclusivos, Guirola et al. ${ }^{18}$ fizeram um estudo randomizado avaliando os resultados do uso de plugs e molas fibradas para a oclusão de veias varicosas e pontos de refluxo. Nesse estudo, o custo médio de cada mola era de cerca de 162 euros e, de cada plug, 880 euros. Ambos os grupos tiveram seguimento de 1, 3, 6 e 12 meses, com sucesso terapêutico avaliado como diminuição ou eliminação dos sintomas. Em ambos os grupos, houve sucesso técnico de $96 \%$ dos casos na embolização das veias-alvo, sendo que, em dois pacientes, não foi possível a embolização da veia ovariana direita por variações anatômicas. Embora o número de dispositivos utilizado tenha sido muito menor no grupo em que se utilizaram plugs, o custo também foi mais elevado nesse grupo. O grupo utilizando molas teve maior número de complicações maiores, com três migrações de molas para artérias pulmonares, enquanto apenas um plug migrou para uma artéria pulmonar direita. Concluíram os autores que, tanto o uso de plugs quanto o de molas são eficazes para a oclusão dos pontos de refluxo e das veias varicosas, com a melhora significativa dos sintomas pélvicos da CVP, com a utilização de menos plugs para a total oclusão dos vasos, com os mesmos resultados clínicos obtidos com as molas fibradas, com tempo de procedimento, de fluoroscopia e doses de radiação também reduzidos ${ }^{18}$. No nosso meio, Siqueira et al. ${ }^{8}$, em estudo retrospectivo de 22 pacientes submetidas à embolização de veias varicosas periuterinas com molas fibradas, observaram melhora clínica em $76,9 \%$ das pacientes, sendo essa melhora ainda mais evidente em pacientes com refluxo grau III da veia ovariana esquerda. Complicações menores, como dor incapacitante, ruptura venosa sem repercussões clínicas e hipotensão postural, foram observadas em $18,2 \%$ dos casos, concluindo os autores que a embolização percutânea é um método eficaz e seguro para o tratamento de pacientes com $\mathrm{CVP}^{19}$.

Embora os dados pareçam corroborar a embolização como tratamento de escolha, a qualidade de evidências é baixa. Daniels et al. ${ }^{19}$, em uma revisão sistemática da literatura utilizando métodos padrão de metanálise, tentaram estimar a proporção geral de pacientes com melhora da sintomatologia após embolização, utilizando as proporções reportadas em estudos individuais. Foram analisados 21 estudos de relatos de casos e um estudo randomizado de baixa qualidade envolvendo 1.308 mulheres. Embora os objetivos e as técnicas de embolização tenham sido claramente detalhados, $1 / 3$ dos estudos não especificou os critérios de avaliação no seguimento e como esses dados seriam coletados. A inclusão apenas de estudos prospectivos não impediu que, em $40 \%$ das publicações, não ficassem claro os critérios pelos quais mulheres foram referendadas para venografia, nem que a perda de seguimento também fosse claramente especificada. Foi relatada melhora inicial importante da dor em cerca de $75 \%$ das pacientes submetidas à embolização com melhora gradual e sustentada com o 
passar do tempo, sendo esse resultado observado em todos os estudos que mensuraram a dor em escalas análogas visuais. No entanto, poucos dados sobre o impacto do tratamento na menstruação, reserva ovariana ou fertilidade foram incluídos. Concluem os autores que se faz necessário um estudo randomizado adequado para corroborar definitivamente a eficácia da embolização no tratamento da CVP'19.

\section{CONCLUSÃO}

A CVP é uma causa frequente de DPC e uma das mais subdiagnosticadas. A valorização dos sintomas durante a anamnese e um exame físico cuidadoso podem levar à suspeita clínica, devendo haver uma correta investigação complementar que inclui exames de imagem para um adequado planejamento terapêutico. Embora os estudos atuais disponíveis contenham fracas evidências devido a desenho inadequado, os dados apresentados e os resultados obtidos parecem corroborar a abordagem endovascular como o tratamento de escolha nesse cenário, permitindo, ao mesmo tempo, eliminar os potenciais pontos de refluxo venoso e a obliteração de veias varicosas, com cessação ou diminuição importante das queixas apresentadas pelas pacientes. Também é de suma importância uma avaliação cuidadosa da anatomia venosa pélvica, haja vista essa ser um fator potencial de morbimortalidade durante intervenções cirúrgicas nesse território anatômico.

\section{REFERÊNCIAS}

1. Zondervan KT, Yudkin PL, Vessey MP, Dawes MG, Barlow DH, Kennedy $\mathrm{SH}$. Prevalence and incidence of chronic pelvic pain in primary care: evidence from a national general practice database. Br J Obstet Gynaecol. 1999;106(11):1149-55. http://dx.doi. org/10.1111/j.1471-0528.1999.tb08140.x. PMid:10549959.

2. Phillips D, Deipolyi AR, Hesketh RL, Midia M, Oklu R. Pelvic congestion syndrome: etiology of pain, diagnosis, and clinical management. J Vasc Interv Radiol. 2014;25(5):725-33. http:// dx.doi.org/10.1016/j.jvir.2014.01.030. PMid:24745902.

3. Chen H, Emura S, Nagasaki S, Kubo KY. Double inferior vena cava with interiliac vein: a case report and literature review. Okajimas Folia Anat Jpn. 2012;88(4):147-51. http://dx.doi.org/10.2535/ ofaj.88.147. PMid:22645906.

4. Hansrani V, Morris J, Caress AL, Payne K, Seif M, McCollum CN. Is pelvic vein incompetence associated with symptoms of chronic pelvic pain in women? A pilot study. Eur J Obstet Gynecol Reprod Biol. 2016;196:21-5. http://dx.doi.org/10.1016/j.ejogrb.2015.10.023. PMid:26656197.

5. Whiteley MS, Lewis-Shiell C, Bishop SI, et al. Pelvic vein embolisation of gonadal and internal iliac veins can be performed safely and with good technical results in an ambulatory vein clinic, under local anaesthetic alone - Results from two years' experience. Phlebology. 2018;33(8):575-9. http://dx.doi.org/10.1177/0268355517734952. PMid:28992753.
6. Borghi C, Dell'Atti L. Pelvic congestion syndrome: the current state of the literature. Arch Gynecol Obstet. 2016;293(2):291-301. http://dx.doi.org/10.1007/s00404-015-3895-7. PMid:26404449.

7. Hansrani V, Abbas A, Bhandari S, Caress AL, Seif M, McCollum $C N$. Trans-venous occlusion of incompetent pelvic veins for chronic pelvic pain in women: a systematic review. Eur J Obstet Gynecol Reprod Biol. 2015;185:156-63. http://dx.doi.org/10.1016/j. ejogrb.2014.12.011. PMid:25590499.

8. Siqueira FM, Monsignore LM, Rosa-E-Silva JC, et al. Evaluation of embolization for periuterine varices involving chronic pelvic pain secondary to pelvic congestion syndrome. Clinics (São Paulo). 2016;71(12):703-8. http://dx.doi.org/10.6061/clinics/2016(12)05. PMid:28076514.

9. Steenbeek MP, van der Vleuten CJM, Schultze Kool LJ, Nieboer TE. Noninvasive diagnostic tools for pelvic congestion syndrome: a systematic review. Acta Obstet Gynecol Scand. 2018;97(7):776-86. http://dx.doi.org/10.1111/aogs.13311. PMid:29381188.

10. Morita S, Higuchi M, Saito N, Mitsuhashi N. Pelvic venous variations in patients with congenital inferior vena cava anomalies: classification with computed tomography. Acta Radiol. 2007;48(9):974-9. http:// dx.doi.org/10.1080/02841850701499409. PMid:17957511.

11. Natsis K, Apostolidis S, Noussios G, Papathanasiou E, Kyriazidou A, Vyzas V. Duplication of the inferior vena cava: anatomy, embryology and classification proposal. Anat Sci Int. 2010;85(1):56-60. http:// dx.doi.org/10.1007/s12565-009-0036-z. PMid:19330283.

12. Spentzouris G, Zandian A, Cesmebasi A, et al. The clinical anatomy of the inferior vena cava: a review of common congenital anomalies and considerations for clinicians. Clin Anat. 2014;27(8):1234-43. http://dx.doi.org/10.1002/ca.22445. PMid:25042045.

13. Beckett D, Dos Santos SJ, Dabbs EB, Shiangoli I, Price BA, Whiteley MS. Anatomical abnormalities of the pelvic venous system and their implications for endovascular management of pelvic venous reflux. Phlebology. 2018;33(8):567-74. http://dx.doi. org/10.1177/0268355517735727. PMid:29059022.

14. Hayashi S, Naito M, Hirai S, et al. Proposal for a new classification of variations in the iliac venous system based on internal iliac veins: a case series and a review of double and left inferior vena cava. Anat Sci Int. 2013;88(4):183-8. http://dx.doi.org/10.1007/ s12565-013-0182-1. PMid:23709295.

15. Tong Z, Gu Y. A case series of duplicated inferior vena cava: mind the side, or fail to trap! Int J Clin Exp Med. 2015;8(5):8029-31. PMid:26221367.

16. Malgor RD, Sobreira M, Boaventura PN, Moura R, Yoshida WB. Implante de filtro em veia cava inferior dupla: relato de caso e revisão da literatura. J Vasc Bras. 2008;7(2):167-70. http://dx.doi. org/10.1590/S1677-54492008000200013.

17. Galego GN, Silveira PG, Bortoluzzi CT, Franklin RN, Ronchi TM. Síndrome da Congestão Venosa Pélvica e resultados do tratamento endovascular: série de casos. J Vasc Bras. 2015;14(3):262-6. http:// dx.doi.org/10.1590/1677-5449.0085.

18. Guirola JA, Sánchez-Ballestin M, Sierre S, Lahuerta C, Mayoral V, De Gregorio MA. A Randomized Trial of Endovascular Embolization Treatment in Pelvic Congestion Syndrome: Fibered Platinum Coils versus Vascular Plugs with 1-Year Clinical Outcomes. J Vasc Interv Radiol. 2018;29(1):45-53. http://dx.doi.org/10.1016/j.jvir.2017.09.011. PMid:29174618.

19. Daniels JP, Champaneria R, Shah L, Gupta JK, Birch J, Moss JG. Effectiveness of embolization or sclerotherapy of pelvic veins for reducing chronic pelvic pain: a systematic review. J Vasc Interv Radiol. 2016;27(10):1478-1486.e8. http://dx.doi.org/10.1016/j. jvir.2016.04.016. PMid:27397619. 
Correspondência Marcelo Fernandes Lima

Rua Marquês de Baependi, Q-B, casa 13 - Condomínio Laranjeiras CEP 69058-130 - Manaus (AM), Brasil

Tel.: (92) 98121-5656 E-mail:mlima_vascular@yahoo.com

Informações sobre os autores MFL - Cirurgião vascular e endovascular, título de especialista pela SBACV/AMB.

IAL - Cirurgião vascular e ultrassonografista vascular, título de especialista pela SBACV/AMB.

VHO - Ginecologista e obstetra, título de especialista pela FEBRASGO; Especialista em Reprodução Humana, Hospital das Clínicas da Faculdade de Medicina da Universidade de São Paulo (HCFMUSP).

Contribuições dos autores Concepção e desenho do estudo: MFL, IAL, VHO Análise e interpretação dos dados: MFL, IAL, VHO Coleta de dados: MFL, IAL, VHO Redação do artigo: MFL Revisão crítica do texto: MFL, IAL, VHO Aprovação final do artigo*: MFL, IAL, VHO Análise estatística: N/A. Responsabilidade geral pelo estudo: MFL

*Todos os autores leram e aprovaram a versão final submetida ao I Vasc Bras. 